\title{
Properties of higher-order half-linear functional differential equations with noncanonical operators
}

\author{
Chenghui Zhang ${ }^{1 *}$, Ravi P Agarwal ${ }^{2}$, Martin Bohner ${ }^{3}$ and Tongxing $\mathrm{Li}^{1}$
}

"Correspondence:
zchui@sdu.edu.cn
${ }^{1}$ School of Control Science and
Engineering, Shandong University,
Jinan, Shandong 250061, P.R. China
Full list of author information is
available at the end of the article

available at the end of the article

\begin{abstract}
Some new results are presented for the oscillatory and asymptotic behavior of higher-order half-linear differential equations with a noncanonical operator. We study the delayed and advanced equations subject to various conditions.

MSC: $34 \mathrm{C} 10 ; 34 \mathrm{~K} 11$

Keywords: asymptotic behavior; oscillation; functional differential equation; higher-order
\end{abstract}

\section{Introduction}

Over the past few years, there has been much research activity concerning the oscillation and asymptotic behavior of various classes of differential equations; we refer the reader to [1-36] and the references cited therein. Half-linear differential equations occur in a variety of real world problems such as in the study of $p$-Laplace equations, non-Newtonian fluid theory, and the turbulent flow of a polytrophic gas in a porous medium; see the related background details reported in [5]. Many authors have studied the properties of solutions of the higher-order differential equation

$$
L x+q(t) x^{\beta}(\tau(t))=0, \quad L x:=\left(r\left(x^{(n-1)}\right)^{\alpha}\right)^{\prime}(t) .
$$

The operator $L x$ is said to be in canonical form if $\int_{t_{0}}^{\infty} r^{-1 / \alpha}(t) \mathrm{d} t=\infty$; otherwise, it is called noncanonical. Throughout the paper, we assume that $\alpha$ and $\beta$ are ratios of odd positive integers, $r \in \mathrm{C}^{1}\left[t_{0}, \infty\right), r(t)>0, r^{\prime}(t) \geq 0, q, \tau \in \mathrm{C}\left[t_{0}, \infty\right), q(t)>0$, and $\lim _{t \rightarrow \infty} \tau(t)=\infty$.

Agarwal et al. [6] established a criterion for the existence of bounded solutions of (1.1) under the assumptions that $n$ is even, $\int_{t_{0}}^{\infty} q(t) \mathrm{d} t=\infty$, and

$$
\int_{t_{0}}^{\infty} r^{-1 / \alpha}(t) \mathrm{d} t<\infty
$$

Zhang et al. [34, 36] obtained some results on asymptotic behavior of (1.1) in the case where (1.2) holds, $\tau(t)<t$, and $\beta \leq \alpha$. In [34,36], an unsolved problem can be formulated as follows.

(P) Is it possible to establish asymptotic criteria for equation (1.1) in the case where $\beta \geq \alpha$ ? 
As a special case when $\alpha=1$ and $n=2$, equation (1.1) becomes

$$
\left(r x^{\prime}\right)^{\prime}(t)+q(t) x^{\beta}(\tau(t))=0
$$

Li et al. [24] established the following criterion for (1.3).

Theorem 1.1 (See [24, Theorem 2.1]) Let (1.2) hold with $\alpha=1, \beta \geq 1, \tau(t) \leq t$, and $\tau^{\prime}(t)>$ 0 for all $t \geq t_{0}$. Assume that there exists a function $\rho \in C^{1}\left(\left[t_{0}, \infty\right), \mathbb{R}\right)$ with $\rho(t) \geq t$ and $\rho^{\prime}(t)>0$ such that, for all sufficiently large $t_{1}$ and for all positive constants $M$ and $L$,

$$
\int^{\infty}\left[q(t) R^{\beta}(\tau(t))-\frac{\beta M^{1-\beta} \tau^{\prime}(t) R^{\beta-1}(\tau(t))}{r(\tau(t)) \int_{t_{1}}^{t} \frac{\tau^{\prime}(s)}{r(\tau(s))} \mathrm{d} s}\right] \mathrm{d} t=\infty
$$

and

$$
\int^{\infty}\left[q(t) \xi^{\beta}(t)-\frac{\beta \rho^{\prime}(t)}{L^{\beta-1} \xi(t) r(\rho(t))}\right] \mathrm{d} t=\infty
$$

where $R(t):=\int_{t_{0}}^{t} r^{-1}(s) \mathrm{d} s$ and $\xi(t):=\int_{\rho(t)}^{\infty} r^{-1}(s) \mathrm{d}$. Then (1.3) is oscillatory.

The purpose of this paper is to solve question ( $\mathrm{P}$ ) and to improve Theorem 1.1. By a solution of equation (1.1) we mean a function $x \in \mathrm{C}^{n-1}\left[T_{x}, \infty\right), T_{x} \geq t_{0}$, which has the property $r\left(x^{(n-1)}\right)^{\alpha} \in \mathrm{C}^{1}\left[T_{x}, \infty\right)$ and satisfies (1.1) on $\left[T_{x}, \infty\right)$. We consider only the solutions satisfying $\sup \{|x(t)|: t \geq T\}>0$ for all $T \geq T_{x}$ and tacitly assume that (1.1) possesses such solutions. A solution of (1.1) is called oscillatory if it has arbitrarily large zeros on $\left[T_{x}, \infty\right)$; otherwise, it is called nonoscillatory. Equation (1.1) is said to be oscillatory if all its solutions are oscillatory.

\section{Main results}

In the sequel, all functional inequalities are assumed to hold eventually, that is, they are satisfied for all $t$ large enough. We use the notation $\delta(t):=\int_{t}^{\infty} r^{-1 / \alpha}(s) \mathrm{d} s$ and $\left(\rho^{\prime}(t)\right)_{+}:=$ $\max \left\{0, \rho^{\prime}(t)\right\}$.

Theorem 2.1 Assume (1.2) and let $n \geq 2, \beta \geq \alpha$, and $\tau(t)<t$ for all $t \geq t_{0}$. Further, assume that the differential equation

$$
y^{\prime}(t)+q(t)\left(\frac{\lambda_{0} \tau^{n-1}(t)}{(n-1) ! r^{1 / \alpha}(\tau(t))}\right)^{\beta} y^{\beta / \alpha}(\tau(t))=0
$$

is oscillatory for some constant $\lambda_{0} \in(0,1)$. If

$$
\begin{aligned}
& \limsup _{t \rightarrow \infty} \int_{t_{0}}^{t}\left[(M \delta(\tau(s)))^{\beta-\alpha} q(s)\left(\frac{\lambda_{1}}{(n-2) !} \tau^{n-2}(s)\right)^{\beta} \delta^{\alpha}(s)\right. \\
& \left.-\frac{\alpha^{\alpha+1}}{(\alpha+1)^{\alpha+1}} \frac{1}{\delta(s) r^{1 / \alpha}(s)}\right] \mathrm{d} s=\infty
\end{aligned}
$$

holds for some constant $\lambda_{1} \in(0,1)$ and for all constants $M>0$, then every solution of (1.1) is oscillatory or tends to zero as $t \rightarrow \infty$. 
Proof Assume that (1.1) has a nonoscillatory solution $x$. Without loss of generality, we may assume that $x$ is eventually positive. Moreover, suppose that $\lim _{t \rightarrow \infty} x(t) \neq 0$. It follows from (1.1) that there exist two possible cases:

(1) $x(t)>0, x^{(n-1)}(t)>0, x^{(n)}(t)<0,\left(r\left(x^{(n-1)}\right)^{\alpha}\right)^{\prime}(t)<0$;

(2) $x(t)>0, x^{(n-2)}(t)>0, x^{(n-1)}(t)<0,\left(r\left(x^{(n-1)}\right)^{\alpha}\right)^{\prime}(t)<0$

for $t \geq t_{1}$, where $t_{1} \geq t_{0}$ is large enough.

Assume that case (1) holds. From [36, Lemma 2.1], we have

$$
x(t) \geq \frac{\lambda t^{n-1}}{(n-1) ! r^{1 / \alpha}(t)}\left(r^{1 / \alpha} x^{(n-1)}\right)(t)
$$

for every $\lambda \in(0,1)$ and for all sufficiently large $t$. Hence by $(1.1)$, we see that $y:=r\left(x^{(n-1)}\right)^{\alpha}$ is a positive solution of the differential inequality

$$
y^{\prime}(t)+q(t)\left(\frac{\lambda \tau^{n-1}(t)}{(n-1) ! r^{1 / \alpha}(\tau(t))}\right)^{\beta} y^{\beta / \alpha}(\tau(t)) \leq 0 .
$$

Using [28, Theorem 1], we see that equation (2.1) also has a positive solution, which is a contradiction.

Assume that case (2) holds. Define the function $w$ by

$$
w(t):=\frac{r(t)\left(x^{(n-1)}\right)^{\alpha}(t)}{\left(x^{(n-2)}\right)^{\alpha}(t)}, \quad t \geq t_{1} .
$$

Then $w(t)<0$ for $t \geq t_{1}$. Noting that $r\left(x^{(n-1)}\right)^{\alpha}$ is decreasing, we have

$$
r^{1 / \alpha}(s) x^{(n-1)}(s) \leq r^{1 / \alpha}(t) x^{(n-1)}(t), \quad s \geq t \geq t_{1} .
$$

Dividing the above inequality by $r^{1 / \alpha}(s)$ and integrating the resulting inequality from $t$ to $l$, we obtain

$$
x^{(n-2)}(l) \leq x^{(n-2)}(t)+r^{1 / \alpha}(t) x^{(n-1)}(t) \int_{t}^{l} \frac{\mathrm{d} s}{r^{1 / \alpha}(s)} .
$$

Letting $l \rightarrow \infty$, we get

$$
x^{(n-2)}(t) \geq-r^{1 / \alpha}(t) x^{(n-1)}(t) \delta(t),
$$

which yields

$$
-\frac{r^{1 / \alpha}(t) x^{(n-1)}(t)}{x^{(n-2)}(t)} \delta(t) \leq 1 .
$$

Thus, by (2.4), we see that

$$
-w(t) \delta^{\alpha}(t) \leq 1
$$

Differentiating (2.4), we have

$$
w^{\prime}(t)=\frac{\left(r\left(x^{(n-1)}\right)^{\alpha}\right)^{\prime}(t)}{\left(x^{(n-2)}\right)^{\alpha}(t)}-\alpha \frac{r(t)\left(x^{(n-1)}\right)^{\alpha+1}(t)}{\left(x^{(n-2)}\right)^{\alpha+1}(t)} .
$$


It follows from (1.1) and (2.4) that

$$
w^{\prime}(t)=-q(t) \frac{x^{\beta}(\tau(t))}{\left(x^{(n-2)}\right)^{\alpha}(t)}-\alpha \frac{w^{(\alpha+1) / \alpha}(t)}{r^{1 / \alpha}(t)} .
$$

By virtue of (2.5), we have

$$
\left(\frac{x^{(n-2)}}{\delta}\right)^{\prime}(t) \geq 0
$$

On the other hand, by [36, Lemma 2.1], we get

$$
x(t) \geq \frac{\lambda}{(n-2) !} t^{n-2} x^{(n-2)}(t)
$$

for every $\lambda \in(0,1)$ and for all sufficiently large $t$. Then from (2.7), (2.8), and (2.9), there exists a constant $M>0$ such that

$$
\begin{aligned}
w^{\prime}(t) & =-q(t) \frac{x^{\beta}(\tau(t))}{\left(x^{(n-2)}(\tau(t))\right)^{\beta}}\left(x^{(n-2)}(\tau(t))\right)^{\beta-\alpha} \frac{\left(x^{(n-2)}(\tau(t))\right)^{\alpha}}{\left(x^{(n-2)}(t)\right)^{\alpha}}-\alpha \frac{w^{(\alpha+1) / \alpha}(t)}{r^{1 / \alpha}(t)} \\
& \leq-(M \delta(\tau(t)))^{\beta-\alpha} q(t)\left(\frac{\lambda}{(n-2) !} \tau^{n-2}(t)\right)^{\beta}-\alpha \frac{w^{(\alpha+1) / \alpha}(t)}{r^{1 / \alpha}(t)} .
\end{aligned}
$$

Multiplying (2.10) by $\delta^{\alpha}(t)$ and integrating the resulting inequality from $t_{1}$ to $t$, we have

$$
\begin{aligned}
& \delta^{\alpha}(t) w(t)-\delta^{\alpha}\left(t_{1}\right) w\left(t_{1}\right)+\alpha \int_{t_{1}}^{t} r^{-1 / \alpha}(s) \delta^{\alpha-1}(s) w(s) \mathrm{d} s \\
& +\int_{t_{1}}^{t}(M \delta(\tau(s)))^{\beta-\alpha} q(s)\left(\frac{\lambda}{(n-2) !} \tau^{n-2}(s)\right)^{\beta} \delta^{\alpha}(s) \mathrm{d} s \\
& +\alpha \int_{t_{1}}^{t} \frac{w^{(\alpha+1) / \alpha}(s)}{r^{1 / \alpha}(s)} \delta^{\alpha}(s) \mathrm{d} s \leq 0 .
\end{aligned}
$$

Set $B:=r^{-1 / \alpha}(s) \delta^{\alpha-1}(s), A:=\delta^{\alpha}(s) / r^{1 / \alpha}(s)$, and $v:=-w(s)$. Using (2.6) and the inequality

$$
A v^{(\alpha+1) / \alpha}-B v \geq-\frac{\alpha^{\alpha}}{(\alpha+1)^{\alpha+1}} \frac{B^{\alpha+1}}{A^{\alpha}}, \quad A>0,
$$

we have

$$
\begin{gathered}
\int_{t_{1}}^{t}\left[(M \delta(\tau(s)))^{\beta-\alpha} q(s)\left(\frac{\lambda}{(n-2) !} \tau^{n-2}(s)\right)^{\beta} \delta^{\alpha}(s)\right. \\
\left.-\frac{\alpha^{\alpha+1}}{(\alpha+1)^{\alpha+1}} \frac{1}{\delta(s) r^{1 / \alpha}(s)}\right] \mathrm{d} s \leq \delta^{\alpha}\left(t_{1}\right) w\left(t_{1}\right)+1,
\end{gathered}
$$

which contradicts (2.2). This completes the proof.

Applying the result of [30] to equation (2.1), we have the following result due to Theorem 2.1 . 
Corollary 2.2 Assume (1.2) and let $n \geq 2, \beta>\alpha, \tau(t)<t$, and $\tau^{\prime}(t)>0$ for all $t \geq t_{0}$. Moreover, assume that there exists a continuously differentiable function $\varphi$ such that

$$
\begin{aligned}
& \varphi^{\prime}(t)>0 \quad \text { and } \quad \lim _{t \rightarrow \infty} \varphi(t)=\infty, \\
& \limsup _{t \rightarrow \infty} \frac{\varphi^{\prime}(\tau(t)) \tau^{\prime}(t)}{\varphi^{\prime}(t)}<\frac{\alpha}{\beta},
\end{aligned}
$$

and

$$
\liminf _{t \rightarrow \infty} \frac{q(t)\left(\frac{\tau^{n-1}(t)}{r^{1 / \alpha}(\tau(t))}\right)^{\beta} \mathrm{e}^{-\varphi(t)}}{\varphi^{\prime}(t)}>0 .
$$

If (2.2) holds for some constant $\lambda_{1} \in(0,1)$ and for all constants $M>0$, then every solution of (1.1) is oscillatory or tends to zero as $t \rightarrow \infty$.

In the following, we establish some results for (1.1) when $n \geq 2$ is even.

Theorem 2.3 Assume (1.2) and let $n \geq 2$ be even, $\beta \geq \alpha, \tau^{\prime}(t)>0$, and $\tau(t) \leq t$ for all $t \geq t_{0}$. Further, assume that there exists a function $\rho \in \mathrm{C}^{1}\left(\left[t_{0}, \infty\right),(0, \infty)\right)$ such that

$$
\limsup _{t \rightarrow \infty} \int_{t_{0}}^{t}\left[K^{\beta-\alpha} q(s) \rho(s)-\frac{1}{(\alpha+1)^{\alpha+1}} \frac{(2(n-2) !)^{\alpha} r(s)\left(\left(\rho^{\prime}(s)\right)_{+}\right)^{\alpha+1}}{\left(\theta_{1} \tau^{\prime}(s) \tau^{n-2}(s) \rho(s)\right)^{\alpha}}\right] \mathrm{d} s=\infty
$$

holds for all constants $\theta_{1} \in(0,1)$ and $K>0$. If $(2.2)$ holds for some constant $\lambda_{1} \in(0,1)$ and for all constants $M>0$, then every solution of (1.1) is oscillatory or tends to zero as $t \rightarrow \infty$.

Proof Assume that (1.1) has a nonoscillatory solution $x$. Without loss of generality, we may assume that $x$ is eventually positive. Moreover, suppose that $\lim _{t \rightarrow \infty} x(t) \neq 0$. It follows from (1.1) that there exist two possible cases (1) and (2) (as those of the proof of Theorem 2.1).

Assume that case (1) holds. From [4, Lemma 2.1], we see that $x^{\prime}(t)>0$ for $t \geq t_{1}$. Define the function $u$ by

$$
u(t):=\rho(t) \frac{r(t)\left(x^{(n-1)}\right)^{\alpha}(t)}{x^{\alpha}(\tau(t) / 2)}, \quad t \geq t_{1}
$$

Then $u(t)>0$ for $t \geq t_{1}$ and

$$
u^{\prime}(t)=\frac{\rho^{\prime}(t)}{\rho(t)} u(t)+\rho(t) \frac{\left(r\left(x^{(n-1)}\right)^{\alpha}\right)^{\prime}(t)}{x^{\alpha}(\tau(t) / 2)}-\alpha \frac{\rho(t) \tau^{\prime}(t)}{2} \frac{r(t)\left(x^{(n-1)}\right)^{\alpha}(t) x^{\prime}(\tau(t) / 2)}{x^{\alpha+1}(\tau(t) / 2)} .
$$

From [4, Lemma 2.2], there exist a $t_{2} \geq t_{1}$ and a constant $\theta_{1}$ with $0<\theta_{1}<1$ such that

$$
x^{\prime}(\tau(t) / 2) \geq \frac{\theta_{1}}{(n-2) !} \tau^{n-2}(t) x^{(n-1)}(t)
$$

for all $t \geq t_{2}$. It follows from (1.1), (2.16), (2.17), and (2.18) that

$$
u^{\prime}(t) \leq \frac{\rho^{\prime}(t)}{\rho(t)} u(t)-q(t) \rho(t) \frac{x^{\beta}(\tau(t))}{x^{\alpha}(\tau(t) / 2)}-\frac{\alpha \tau^{\prime}(t)}{2} \frac{\theta_{1}}{(n-2) !} \tau^{n-2}(t) \frac{u^{(\alpha+1) / \alpha}(t)}{(\rho(t) r(t))^{1 / \alpha}} .
$$


Using $x^{\prime}>0$ and (2.19), we get

$$
\begin{aligned}
u^{\prime}(t) \leq & \frac{\left(\rho^{\prime}(t)\right)_{+}}{\rho(t)} u(t)-K^{\beta-\alpha} q(t) \rho(t) \\
& -\frac{\alpha \tau^{\prime}(t)}{2} \frac{\theta_{1}}{(n-2) !} \tau^{n-2}(t) \frac{u^{(\alpha+1) / \alpha}(t)}{(\rho(t) r(t))^{1 / \alpha}}
\end{aligned}
$$

for some constant $K>0$. Set

$$
A:=\frac{\alpha \tau^{\prime}(t)}{2} \frac{\theta_{1}}{(n-2) !} \frac{\tau^{n-2}(t)}{(\rho(t) r(t))^{1 / \alpha}}, \quad B:=\frac{\left(\rho^{\prime}(t)\right)_{+}}{\rho(t)}, \quad \text { and } \quad v:=u(t)
$$

Using inequality (2.11), we obtain

$$
\begin{aligned}
& \frac{\left(\rho^{\prime}(t)\right)_{+}}{\rho(t)} u(t)-\frac{\alpha \tau^{\prime}(t)}{2} \frac{\theta_{1}}{(n-2) !} \tau^{n-2}(t) \frac{u^{(\alpha+1) / \alpha}(t)}{(\rho(t) r(t))^{1 / \alpha}} \\
& \quad \leq \frac{1}{(\alpha+1)^{\alpha+1}} \frac{(2(n-2) !)^{\alpha} r(t)\left(\left(\rho^{\prime}(t)\right)_{+}\right)^{\alpha+1}}{\left(\theta_{1} \tau^{\prime}(t) \tau^{n-2}(t) \rho(t)\right)^{\alpha}} .
\end{aligned}
$$

Substituting the last inequality into (2.20), we get

$$
u^{\prime}(t) \leq-K^{\beta-\alpha} q(t) \rho(t)+\frac{1}{(\alpha+1)^{\alpha+1}} \frac{(2(n-2) !)^{\alpha} r(t)\left(\left(\rho^{\prime}(t)\right)_{+}\right)^{\alpha+1}}{\left(\theta_{1} \tau^{\prime}(t) \tau^{n-2}(t) \rho(t)\right)^{\alpha}} .
$$

Integrating (2.21) from $t_{2}$ to $t$, we have

$$
\int_{t_{2}}^{t}\left[K^{\beta-\alpha} q(s) \rho(s)-\frac{1}{(\alpha+1)^{\alpha+1}} \frac{(2(n-2) !)^{\alpha} r(s)\left(\left(\rho^{\prime}(s)\right)_{+}\right)^{\alpha+1}}{\left(\theta_{1} \tau^{\prime}(s) \tau^{n-2}(s) \rho(s)\right)^{\alpha}}\right] \mathrm{d} s \leq u\left(t_{2}\right),
$$

which contradicts (2.15). Assume that case (2) holds. Proceeding as in the proof of Theorem 2.1, we can obtain a contradiction to (2.2). This completes the proof.

Next we establish a result for (1.1) when $n=2$.

Theorem 2.4 Assume (1.2) and let $n=2, \beta \geq \alpha, \tau^{\prime}(t)>0$, and $\tau(t) \leq t$ for $t \geq t_{0}$. Further, assume that there exists a function $\rho \in \mathrm{C}^{1}\left(\left[t_{0}, \infty\right),(0, \infty)\right)$ such that

$$
\limsup _{t \rightarrow \infty} \int_{t_{0}}^{t}\left[K^{\beta-\alpha} q(s) \rho(s)-\frac{1}{(\alpha+1)^{\alpha+1}} \frac{r(s)\left(\left(\rho^{\prime}(s)\right)_{+}\right)^{\alpha+1}}{\left(\tau^{\prime}(s) \rho(s)\right)^{\alpha}}\right] \mathrm{d} s=\infty
$$

for all constants $K>0$. If

$$
\limsup _{t \rightarrow \infty} \int_{t_{0}}^{t}\left[(M \delta(\tau(s)))^{\beta-\alpha} q(s) \delta^{\alpha}(s)-\frac{\alpha^{\alpha+1}}{(\alpha+1)^{\alpha+1}} \frac{1}{\delta(s) r^{1 / \alpha}(s)}\right] \mathrm{d} s=\infty
$$

holds for all constants $M>0$, then (1.1) is oscillatory.

Proof Assume that (1.1) has a nonoscillatory solution $x$. Without loss of generality, we may assume that $x$ is eventually positive. It follows from (1.1) that there exist two possible cases 
(1) and (2) with $n=2$ (as those of the proof of Theorem 2.1). Assume that case (1) holds. Define

$$
u(t):=\rho(t) \frac{r(t)\left(x^{\prime}(t)\right)^{\alpha}}{x^{\alpha}(\tau(t))}, \quad t \geq t_{1}
$$

The rest of the proof is similar to that of Theorem 2.3, and so is omitted. Assume that case (2) holds. Similar as in the proof of Theorem 2.1, we can obtain a contradiction to (2.23). This completes the proof.

Next we establish some oscillation criteria for (1.1) when $n \geq 2$ is even and $\tau(t)>t$ for all $t \geq t_{0}$.

Theorem 2.5 Assume (1.2) and let $n \geq 2$ be even, $\beta>\alpha$, and $\tau(t)>t$ for all $t \geq t_{0}$. Further, assume that there exists a function $\rho \in \mathrm{C}^{1}\left(\left[t_{0}, \infty\right),(0, \infty)\right)$ such that

$$
\limsup _{t \rightarrow \infty} \int_{t_{0}}^{t}\left[K^{\beta-\alpha} q(s) \rho(s)-\frac{1}{(\alpha+1)^{\alpha+1}} \frac{(2(n-2) !)^{\alpha} r(s)\left(\left(\rho^{\prime}(s)\right)_{+}\right)^{\alpha+1}}{\left(\theta_{1} s^{n-2} \rho(s)\right)^{\alpha}}\right] \mathrm{d} s=\infty
$$

holds for all constants $\theta_{1} \in(0,1)$ and $K>0$. If

$$
\int^{\infty} q(t) \delta^{\beta}(\tau(t))\left(\tau^{n-2}(t)\right)^{\beta} \mathrm{d} t=\infty
$$

then every solution of (1.1) is oscillatory or tends to zero as $t \rightarrow \infty$.

Proof Assume that (1.1) has a nonoscillatory solution $x$. Without loss of generality, we may assume that $x$ is eventually positive. Moreover, suppose that $\lim _{t \rightarrow \infty} x(t) \neq 0$. It follows from (1.1) that there exist two possible cases (1) and (2) (as those of the proof of Theorem 2.1).

Assume that case (1) holds. From [4, Lemma 2.1], we see that $x^{\prime}(t)>0$ for $t \geq t_{1}$. Define the function $u$ by

$$
u(t):=\rho(t) \frac{r(t)\left(x^{(n-1)}\right)^{\alpha}(t)}{x^{\alpha}(t / 2)}, \quad t \geq t_{1}
$$

Then $u(t)>0$ for $t \geq t_{1}$ and

$$
u^{\prime}(t)=\frac{\rho^{\prime}(t)}{\rho(t)} u(t)+\rho(t) \frac{\left(r\left(x^{(n-1)}\right)^{\alpha}\right)^{\prime}(t)}{x^{\alpha}(t / 2)}-\alpha \frac{\rho(t)}{2} \frac{r(t)\left(x^{(n-1)}\right)^{\alpha}(t) x^{\prime}(t / 2)}{x^{\alpha+1}(t / 2)} .
$$

From [4, Lemma 2.2], there exist a $t_{2} \geq t_{1}$ and a constant $\theta_{1}$ with $0<\theta_{1}<1$ such that

$$
x^{\prime}(t / 2) \geq \frac{\theta_{1}}{(n-2) !} t^{n-2} x^{(n-1)}(t)
$$

for all $t \geq t_{2}$. Thus

$$
u^{\prime}(t) \leq \frac{\rho^{\prime}(t)}{\rho(t)} u(t)-q(t) \rho(t) \frac{x^{\beta}(\tau(t))}{x^{\alpha}(t / 2)}-\frac{\alpha}{2} \frac{\theta_{1}}{(n-2) !} t^{n-2} \frac{u^{(\alpha+1) / \alpha}(t)}{(\rho(t) r(t))^{1 / \alpha}} .
$$


Similar as in the proof of Theorem 2.3, we can get a contradiction to (2.24). Assume that case (2) holds. We have (2.5) and (2.9) for every $\lambda \in(0,1)$ and for all sufficiently large $t$. Thus, we get by (1.1), (2.5), and (2.9) that

$$
\left(r\left(x^{(n-1)}\right)^{\alpha}\right)^{\prime}(t)-q(t)\left(\frac{\lambda}{(n-2) !} \tau^{n-2}(t) \delta(\tau(t))\right)^{\beta}\left(r^{1 / \alpha} x^{(n-1)}\right)^{\beta}(\tau(t)) \leq 0 .
$$

Let $u:=r\left(x^{(n-1)}\right)^{\alpha}$. Then $y:=-u>0$ is a solution of the advanced inequality

$$
y^{\prime}(t)-q(t)\left(\frac{\lambda}{(n-2) !} \tau^{n-2}(t) \delta(\tau(t))\right)^{\beta} y^{\beta / \alpha}(\tau(t)) \geq 0 .
$$

It follows from [8, Lemma 2.3] that the corresponding advanced differential equation

$$
y^{\prime}(t)-q(t)\left(\frac{\lambda}{(n-2) !} \tau^{n-2}(t) \delta(\tau(t))\right)^{\beta} y^{\beta / \alpha}(\tau(t))=0
$$

has an eventually positive solution. Using condition (2.25) and [22, Theorem 1], one can obtain a contradiction. This completes the proof.

Finally, we establish a result for (1.1) when $n=2$.

Theorem 2.6 Assume (1.2) and let $n=2, \beta>\alpha$, and $\tau(t)>t$ for $t \geq t_{0}$. Further, assume that there exists a function $\rho \in \mathrm{C}^{1}\left(\left[t_{0}, \infty\right),(0, \infty)\right)$ such that

$$
\limsup _{t \rightarrow \infty} \int_{t_{0}}^{t}\left[K^{\beta-\alpha} q(s) \rho(s)-\frac{1}{(\alpha+1)^{\alpha+1}} \frac{r(s)\left(\left(\rho^{\prime}(s)\right)_{+}\right)^{\alpha+1}}{\rho^{\alpha}(s)}\right] \mathrm{d} s=\infty
$$

for all constants $K>0$. If

$$
\int^{\infty} q(t) \delta^{\beta}(\tau(t)) \mathrm{d} t=\infty
$$

then (1.1) is oscillatory.

Proof Assume that (1.1) has a nonoscillatory solution $x$. Without loss of generality, we may assume that $x$ is eventually positive. It follows from (1.1) that there exist two possible cases (1) and (2) with $n=2$ (as those of the proof of Theorem 2.1). Assume that case (1) holds. Define

$$
u(t):=\rho(t) \frac{r(t)\left(x^{\prime}(t)\right)^{\alpha}}{x^{\alpha}(t)}, \quad t \geq t_{1} .
$$

The rest of the proof is similar to that of Theorem 2.3, and so is omitted. Assume that case (2) holds. Similar as in the proof of Theorem 2.5, we can obtain a contradiction to (2.27). This completes the proof.

\section{Examples and discussions}

In the following, we illustrate possible applications with two examples. 
Example 3.1 For $t \geq 1$, consider the second-order delay differential equation

$$
\left(\mathrm{e}^{t} x^{\prime}(t)\right)^{\prime}+\mathrm{e}^{5 t / 2} x^{3}\left(\frac{t}{2}\right)=0
$$

Let $\alpha=1, \beta=3$, and $\rho(t)=1$. Note that $\delta(t)=\mathrm{e}^{-t}$. Using Theorem 2.4, equation (3.1) is oscillatory. It is not difficult to see that Theorem 1.1 fails to apply due to condition (1.4).

Example 3.2 For $t \geq 1$, consider the second-order advanced differential equation

$$
\left(\mathrm{e}^{t} x^{\prime}(t)\right)^{\prime}+\frac{\mathrm{e}^{6 t}}{t} x^{3}(2 t)=0
$$

Let $\alpha=1, \beta=3$, and $\rho(t)=1$. Note that $\delta(t)=\mathrm{e}^{-t}$. Using Theorem 2.6, equation (3.2) is oscillatory.

In this paper, we suggested some new results on the oscillation and asymptotic behavior of differential equation (1.1). Theorem 2.1 can be applied in the odd-order and even-order equations.

We stress that the study of equation (1.1) in the case (1.2) brings additional difficulties. Since the sign of $x^{(n-1)}$ is not known, our criteria include a pair of assumptions; see, e.g., (2.2) and (2.15). We utilized two different methods (Riccati substitution and comparison method) to deal with the cases $\tau(t) \leq t$ and $\tau(t)>t$.

Competing interests

The authors declare that they have no competing interests.

\section{Authors' contributions}

All authors read and approved the final manuscript.

\section{Author details}

${ }^{1}$ School of Control Science and Engineering, Shandong University, Jinan, Shandong 250061, P.R. China. ${ }^{2}$ Department of Mathematics, Texas A\&M University-Kingsville, 700 University Blvd., Kingsville, TX 78363-8202, USA. ${ }^{3}$ Department of Mathematics and Statistics, Missouri S\&T, Rolla, MO 65409-0020, USA.

\section{Acknowledgements}

This research is supported by NNSF of P.R. China (Grant Nos. 61034007, 51277116, 50977054).

Received: 4 December 2012 Accepted: 7 February 2013 Published: 12 March 2013

\section{References}

1. Agarwal, RP, Bohner, M, Li, W: Nonoscillation and Oscillation: Theory for Functional Differential Equations. Monographs and Textbooks in Pure and Applied Mathematics, vol. 267. Marcel Dekker, New York (2004)

2. Agarwal, RP, Grace, SR: Oscillation of certain functional differential equations. Comput. Math. Appl. 38, 143-153 (1999)

3. Agarwal, RP, Grace, SR, O'Regan, D: Oscillation Theory for Difference and Functional Differential Equations. Kluwer Academic, Dordrecht (2000)

4. Agarwal, RP, Grace, SR, O'Regan, D: Oscillation criteria for certain $n$th order differential equations with deviating arguments. J. Math. Anal. Appl. 262, 601-622 (2001)

5. Agarwal, RP, Grace, SR, O'Regan, D: Oscillation Theory for Second Order Linear, Half-Linear, Superlinear and Sublinear Dynamic Equations. Kluwer Academic, Dordrecht (2002)

6. Agarwal, RP, Grace, SR, O'Regan, D: The oscillation of certain higher-order functional differential equations. Math. Comput. Model. 37, 705-728 (2003)

7. Agarwal, RP, Shieh, S-L, Yeh, C-C: Oscillation criteria for second-order retarded differential equations. Math. Comput. Model. 26, 1-11 (1997)

8. Baculíková, B: Properties of third-order nonlinear functional differential equations with mixed arguments. Abstr. Appl. Anal. 2011, 1-15 (2011)

9. Baculíková, B, Džurina, J: Oscillation of third-order nonlinear differential equations. Appl. Math. Lett. 24, 466-470 (2011)

10. Baculíková, B, Džurina, J: Oscillation of third-order functional differential equations. Electron. J. Qual. Theory Differ. Equ. 43, 1-10 (2010) 
11. Baculíková, B, Džurina, J, Graef, JR: On the oscillation of higher order delay differential equations. Nonlinear Oscil. 15 13-24 (2012)

12. Dahiya, RS: Oscillation criteria of even-order nonlinear delay differential equations. J. Math. Anal. Appl. 54, 653-665 (1976)

13. Džurina, J, Baculíková, B: Oscillation and asymptotic behavior of higher-order nonlinear differential equations. Int. J. Math. Math. Sci. 2012, 1-9 (2012)

14. Džurina, J, Stavroulakis, IP: Oscillation criteria for second order delay differential equations. Appl. Math. Comput. 140 445-453 (2003)

15. Erbe, L, Kong, Q, Zhang, B: Oscillation Theory for Functional Differential Equations. Marcel Dekker, New York (1995)

16. Grace, SR: Oscillation theorems for $n$ th-order differential equations with deviating arguments. J. Math. Anal. Appl. 101, 268-296 (1984)

17. Grace, SR: Oscillation theorems for certain functional differential equations. J. Math. Anal. Appl. 184, 100-111 (1994)

18. Grace, SR, Agarwal, RP, Pavani, R, Thandapani, E: On the oscillation of certain third order nonlinear functional differential equations. Appl. Math. Comput. 202, 102-112 (2008)

19. Grace, SR, Lalli, BS: Oscillation theorems for nth-order delay differential equations. J. Math. Anal. Appl. 91, 352-366 (1983)

20. Grace, SR, Lalli, BS: Oscillation of even order differential equations with deviating arguments. J. Math. Anal. Appl. 147, 569-579 (1990)

21. Kartsatos, AG: On oscillation of solutions of even order nonlinear differential equations. J. Differ. Equ. 6, 232-237 (1969)

22. Kitamura, Y, Kusano, T: Oscillation of first-order nonlinear differential equations with deviating arguments. Proc. Am. Math. Soc. 78, 64-68 (1980)

23. Ladde, GS, Lakshmikantham, V, Zhang, BG: Oscillation Theory of Differential Equations with Deviating Arguments. Marcel Dekker, New York (1987)

24. Li, T, Han, Z, Zhang, C, Sun, S: On the oscillation of second-order Emden-Fowler neutral differential equations. J. Appl. Math. Comput. 37, 601-610 (2011)

25. $\mathrm{Li}, \mathrm{T}$, Thandapani, E: Oscillation of solutions to odd-order nonlinear neutral functional differential equations. Electron. J. Differ. Equ. 23, 1-12 (2011)

26. Mahfoud, WE: Oscillation and asymptotic behavior of solutions of $n$th order nonlinear delay differential equations. J. Differ. Equ. 24, 75-98 (1977)

27. Philos, CG: A new criterion for the oscillatory and asymptotic behavior of delay differential equations. Bull. Acad. Pol. Sci., Sér. Sci. Math. 39, 61-64 (1981)

28. Philos, CG: On the existence of nonoscillatory solutions tending to zero at $\infty$ for differential equations with positive delays. Arch. Math. 36, 168-178 (1981)

29. Rogovchenko, YV, Tuncay, F: Oscillation theorems for a class of second order nonlinear differential equations with damping. Taiwan. J. Math. 13, 1909-1928 (2009)

30. Tang, X: Oscillation for first order superlinear delay differential equations. J. Lond. Math. Soc. 65, 115-122 (2002)

31. $\mathrm{Xu}, \mathrm{Z}, \mathrm{Xia}, \mathrm{Y}$ : Integral averaging technique and oscillation of certain even order delay differential equations. J. Math. Anal. Appl. 292, 238-246 (2004)

32. Yildiz, MK, Öcalan, Ö: Oscillation results of higher order nonlinear neutral delay differential equations. Selçuk J. Appl. Math. 11, 55-62 (2010)

33. Zhang, B: Oscillation of even order delay differential equations. J. Math. Anal. Appl. 127, 140-150 (1987)

34. Zhang, C, Agarwal, RP, Bohner, M, Li, T: New results for oscillatory behavior of even-order half-linear delay differential equations. Appl. Math. Lett. 26, 179-183 (2013)

35. Zhang, C, Agarwal, RP, Bohner, M, Li, T: Oscillation of third-order nonlinear delay differential equations. Taiwan J. Math. 17(2), 545-558 (2013)

36. Zhang, C, Li, T, Sun, Bo, Thandapani, E: On the oscillation of higher-order half-linear delay differential equations. Appl. Math. Lett. 24, 1618-1621 (2011)

doi:10.1186/1687-1847-2013-54

Cite this article as: Zhang et al.: Properties of higher-order half-linear functional differential equations with noncanonical operators. Advances in Difference Equations 2013 2013:54.

\section{Submit your manuscript to a SpringerOpen ${ }^{\ominus}$ journal and benefit from:}

- Convenient online submission

Rigorous peer review

Immediate publication on acceptance

- Open access: articles freely available online

- High visibility within the field

- Retaining the copyright to your article 